\title{
Reactivity of Thinking Aloud in EFL Learners' Performance in Vocabulary Tests
}

\author{
Yuko HOSHINO
}

Tokyo Fuji University

\begin{abstract}
This study examines the effects of reactivity in a vocabulary test in sentential context by comparing the performance of two groups: one using thinking aloud and the other not using thinking aloud. Although using thinking aloud in investigating the learning process is a widespread practice, there is a possibility that conducting thinking aloud might change the learner's cognitive process; hence, the data might not reflect the true process. The results of comparing two groups, one using thinking aloud and the other not using it, showed that the thinking-aloud group outperformed the control group. There were two possible interpretations: the thinking aloud itself affected the test performance, or other variables accompanying the thinking aloud were factors that caused the improved test score. The results of further analysis showed that the difference of the time allotment between the two groups rather than the characteristics of thinking aloud could influence the test performance. From these results, this study adds further proof of the validity of using thinking aloud as a methodology for collecting data about test-taking processes.
\end{abstract}

\section{Introduction}

When conducting any kind of test, the test researchers are interested in the process of taking the test as well as the product. It is rather easy to investigate the product because we can see it through the test results. On the other hand, it is quite difficult to examine the process because we need to consider what the testees are thinking and doing while they take the test. Among the many methods for investigating the test-taking processes that have been devised and adopted, thinking aloud is one of the most popular. It is used not only to investigate test-taking processes (e.g., Cohen \& Upton, 2007; Nikolov, 2006) but also to clarify reading processes (e.g., Kavale \& Schreiner, 1979), reading strategies (e.g., Block, 1986), and processes for summary writing (e.g., Brown \& Day, 1983).

All the methodologies have both good and bad points, and this is also the case for thinking aloud. What Takač (2008) suggests is we can obtain direct online information through thinking aloud, but it is difficult to interpret and generalize the data and we cannot identify everything the participants do when they tackle the task because they do 
not verbalize what they do subconsciously. Moreover, thinking aloud itself might change the task performance, which is a great concern for test researchers. If test performance under the thinking aloud condition is different from that under the ordinary testing condition (i.e., without thinking aloud), the observed behavior and collected verbal data do not provide useful information about the test-taking processes in the normal condition, therefore, the validity of using thinking aloud is impaired. The change in task performance caused by thinking-aloud is called reactivity, and it raises the issue of "thinking aloud potentially triggering changes in learners' cognitive processes while performing the task" (Leow \& Morgan-Short, 2004, p. 35). Reactivity has been investigated mainly outside the field of second language acquisition (e.g., Biggs, Rosman, \& Serfenian, 1993; Russo, Johnson, \& Stephens, 1989), and such studies have found that there was no reactivity, even though the time taken in the task increased when adopting thinking-aloud. In SLA, Leow and Morgan-Short examined the effect of thinking aloud on reading tasks. As with other studies, they did not find a significant difference between the think-aloud group and the non-think-aloud group. However, the number of studies on reactivity is still small, especially in the SLA area, and it is necessary to investigate whether there is reactivity in other tests apart from reading tests. Hence, this study uses vocabulary tests and examines the reactivity further by comparing the performance of two groups: one using thinking aloud and the other not using thinking aloud. The research question addressed in this study is as follows:

RQ: Is there any evidence of reactivity in Japanese EFL learners' performance in vocabulary tests presented in context?

\section{Method}

\subsection{Participants}

There were two types of participants: those who thought aloud while taking the test and those who did not. In terms of the former, there were 20 participants from a single university, and they were all paid volunteers. Their majors were diverse, including health and physical education, knowledge and library sciences, policy and planning sciences, mathematics, physics, life and environmental sciences, and chemistry.

As for the latter participants, 143 students took part in the study. Their majors were Japanese, Chinese, German, health and physical education, knowledge and library sciences, policy and planning sciences, life and environmental sciences, comparative culture, engineering sciences, humanities, mathematics, physics, and chemistry. They 
were university students from the same university as those who thought aloud.

\subsection{Materials}

Two kinds of test were used: the vocabulary proficiency test and the experimental test. In the vocabulary proficiency test, a total of 28 items were used from the vocabulary and grammar sections in the 2nd, pre-2nd, and 3rd grades of the Society for Testing English Proficiency test conducted in January, 2005. The test formats were multiple-choice (MC) tests in context with supplying-format. Originally there were 55 items in total, but items measuring grammatical knowledge were excluded after being assessed by two graduate students majoring in English education (Cronbach's $\alpha=1.00$ ): accordingly 44 items remained. After that, 118 university students who did not participate in the main study answered the remaining vocabulary items to select the best items for the vocabulary proficiency test. Misfit items (including underfit and overfit items) were excluded by the rationale of Bond and Fox (2007, p. 240) who determined that mean squares of over 1.3 were underfit, and below 0.75 were overfit. Consequently, 28 items ( 13 in 2 nd, 9 in pre-2nd, and 6 in 3 rd grade) were used as the vocabulary proficiency test.

In terms of the experimental test, 70 original items of $\mathrm{MC}$ vocabulary tests in sentential context were created. The format was almost identical to that of the vocabulary proficiency test. The target words were of high frequency (i.e., levels 1 and 2 from JACET 8000) in order to match the participants' English language ability. From 70 items, the 39 best items were chosen for the main study. These were selected from the viewpoint of discriminability and whether there was one definite answer from four alternatives. Before conducting the experiment, care was taken to ensure that all of the words appeared only once as a choice in order to decrease the possibility of local independency. However, it was impossible to control the frequency of appearance of those words in context because some of the target words were extremely high frequency, such as have, and controlling the frequency of these words would make context unnatural and difficult to understand.

\subsection{Procedure}

Participants who took the tests without thinking aloud answered the vocabulary proficiency test in 15 minutes, and then they took the experimental test in 25 minutes. Participants who were to think aloud answered the vocabulary proficiency test in 15 minutes, and then they were instructed on how to think aloud. This instruction included an explanation of the purpose of this study and how to think aloud, and also involved 
practicing thinking aloud using three items selected from the vocabulary proficiency test. These instructions were based on Cohen and Upton (2006). After confirming they were sure of what to do, they took the experimental test. They were allowed to spend as much time as they wanted, and average time taken was 58 minutes (range: 38 to 76 minutes).

\section{Results and Discussion}

First, reliability, validity and discriminability were examined. Reliability was high for those who did not think aloud ( $n=143$, Cronbach's $\alpha=.83$ for the vocabulary proficiency test and $\alpha=.82$ for the experimental test), but low for those who did think aloud ( $n=21, \alpha=.46$ for the vocabulary proficiency test and $\alpha=.44$ for the experimental test). Since all the participants answered exactly the same tests, this discrepancy arose from smaller $S D$ (see Table 2). It was thought that the reliability coefficients would improve if there was greater variety in proficiency levels among participants who thought aloud, so both tests were regarded as reliable.

Validity was examined using two methods. First, unidimensionality was confirmed through the infit mean square of participants who did not think aloud, because the number of test-takers who thought aloud was too small to use Rasch analysis. The criteria of the infit mean square was the same as 2.2 , using 0.75 to 1.3 by Bond and Fox (2007, p. 240). In both the vocabulary proficiency test and the experimental test, the infit mean square of all the items calculated by Winsteps (Linacre, 2006) was between 0.75 and 1.3; hence, the tests used in this study were regarded as unidimensional. The second method for confirming validity was to calculate correlation coefficients between the vocabulary proficiency test and the experimental test, since both are intended to measure the same construct: vocabulary knowledge in context. The coefficients were $r=.72$ for those who did not think aloud, and $r=.42$ for those who did. The low correlation coefficient for the participants who thought aloud was due to the smaller $S D$ : therefore, the results indicate that both tests were valid.

As for discriminability, Fisher's Z-transformation and its inverse were used. With regard to the vocabulary proficiency test, average point biserial correlation coefficients were .36 for those who did not think aloud and .11 for those who did. As for the experimental test, figures for average discriminability were .30 and .10 , respectively. Differences appeared between the two conditions of participants, again, because of the small number of participants who thought aloud.

Table 1 shows the descriptive statistics of the vocabulary proficiency test and the experimental test. In order to statistically compare the mean scores between the two groups, 20 participants were randomly chosen among the participants who did not think 
aloud. This was because the $t$ test and the $F$ test were sensitive to the different sample sizes of the groups (Glass \& Hopkins, 1996). If we compared the participants directly, leaving aside the great difference in the number of participants, there would be a huge type-I error. The descriptive statistics and the results of the $t$ test are presented in Table 2. The difference between the two groups of participants in the vocabulary proficiency test was not statistically significant but there was a significant tendency for differences to occur between the groups in the experimental test. Therefore, from the results of the vocabulary proficiency test it can be supposed that the two groups of participants had identical vocabulary knowledge. However, when one group conducted thinking aloud, the group's performance could outperform the performance of the other group members who did not think aloud.

Table 1

Descriptive Statistics for the Vocabulary Proficiency Test and the Experimental Test

\begin{tabular}{llrcl}
\hline & & $n$ & $M$ & $S D$ \\
\hline Proficiency Test & Not thinking aloud & 143 & 22.36 & 4.50 \\
& Thinking aloud & 20 & 22.85 & 2.58 \\
\hline \multirow{2}{*}{ Experimental Test } & Not thinking aloud & 143 & 23.69 & 6.23 \\
& Thinking aloud & 20 & 26.20 & 3.41 \\
\hline
\end{tabular}

Table 2

Results of $t$ Tests for the Difference Between Participants Who did and did not Think Aloud

\begin{tabular}{|c|c|c|c|c|c|c|c|}
\hline & \multicolumn{2}{|c|}{ Thinking aloud $(n=20)$} & \multicolumn{2}{|c|}{ Not thinking aloud $(n=20)$} & \multirow[b]{2}{*}{$\stackrel{t}{2}$} & \multirow[b]{2}{*}{$d f$} & \multirow[b]{2}{*}{$p$} \\
\hline & Mean & $S D$ & Mean & $S D$ & & & \\
\hline Proficiency Test & 23.50 & 4.24 & 22.85 & 2.58 & -.59 & 38 & .56 \\
\hline Experimental Test & 23.35 & 6.18 & 26.20 & 3.41 & 1.81 & 38 & .08 \\
\hline
\end{tabular}

The participants who thought aloud obtained higher scores for two possible reasons. The first reason could be the time allotment. The participants who did not think aloud had to finish their tests within 25 minutes, while the participants who thought aloud did not have a time limit. Actually, among the all participants who did not think aloud, two students could not answer the last three items, and four other students failed to answer the last five or six items, although this number was small considering there were 143 participants in total.

The second reason could be the characteristics of the think-aloud protocol. As the 
participants were asked to say what they were thinking, they explained their thoughts or ideas, or sometimes they were asked to state reasons why they had selected one specific choice; and such actions might have a positive influence on test-takers. The study of lexical inferencing by Cain (2007) showed that those who explained the interpretation of novel words or who were provided with an explanation by teachers obtained more accurate definitions of target words than those who just read and inferred the meaning of unknown words. Hence, verbalization itself may improve test performance.

Table 3.

Descriptive Statistics and the Results of a MANOVA for Those Items with Significant Difference or Difference Approaching Significance

\begin{tabular}{lccccccc}
\hline \multicolumn{7}{l}{ Thinking aloud $(n=20)$} & \multicolumn{2}{l}{ Not thinking aloud $(n=20)$} & & & \\
\hline Item & Mean & $S D$ & Mean & $S D$ & $F$ & $d f$ & $p$ \\
\hline 2 & 1.00 & .00 & .60 & .50 & 12.67 & 1 & .001 \\
\hline 30 & .80 & .41 & .50 & .51 & .90 & 1 & .048 \\
\hline 35 & .80 & .41 & .55 & .51 & .63 & 1 & .095 \\
\hline 36 & 1.00 & .00 & .70 & .47 & .90 & 1 & .01 \\
\hline 38 & .75 & .44 & .35 & .49 & 1.60 & 1 & .01 \\
\hline 39 & .55 & .51 & .25 & .44 & .90 & 1 & .06 \\
\hline
\end{tabular}

Note. Means and SDs are proportions.

In order to investigate which possibility would match the interpretation of the current result, a one-way MANOVA was conducted to see in which items the difference appeared. The results showed a significant difference in items $2,30,36$, and 38 , and a significant tendency in items 35 and 39. The descriptive statistics and the results of a MANOVA of these items can be seen in Table 3. In all of the items, the performance of participants who thought aloud surpassed that of participants who did not think aloud. Moreover, most of the items were in the last quarter of the test; hence, the time allotment might have been too short for some participants who did not think aloud. That is, it was possible that they expended too much time on the first three quarters of the items and thus there was too little time left for the latter items. However, the time allotment was decided based on the results of the pilot study and most of the participants who did not think aloud finished answering all the items within the allotted time, so it can be regarded that the majority of the test-takers had enough time to take the tests. Nevertheless, the time taken by the thinking aloud group was much longer (average: 58 minutes) than the non-thinking-aloud group (time allotment: 25 minutes). 
In this sense, this study is different from that of Leow and Morgan-Short (2004). In their study, the think-aloud group took 25 minutes while the non-thinking-aloud group took 20 minutes on average, Therefore, it is quite possible that the difference found in this study was due to the time used for the test, even though only six participants out of 143 could not finish the test in the determined time in the non-thinking-aloud group.

One exception item from the result of the MANOVA above was item 2, which was located at the very beginning of the test. All the participants who thought aloud were correct $(100.00 \%)$, but the proportion correct of those who did not think aloud was $60.00 \%$ (12 out of 20 randomly chosen test-takers), and even considering all the participants (i.e., 143 test-takers), the proportion correct was $67.83 \%$. Among those who got it wrong, only one person selected moments and the others selected period for the answers among the 20 participants who did not think aloud. In order to investigate the reason, the thinking aloud protocol was examined. Although everyone who thought aloud selected the correct answer, two of the participants left period as a possible answer as follows. The protocol related to period was underlined.

Friends like to be given something you've taken (__ ) and trouble to make for them.
a. moments
b. morning
c. period
d. time*

$[\mathrm{ID} 7]^{1}$

"FRIENDS LIKE TO DO SOMETHING, LIKE TO BE GIVEN SOMETHING, YOU ARE... trouble to make for them, TROUBLE, WELL, THE ANSWER IS time, MEANING TAKING TIME. Moments MEAN INSTANT, morning IS MORNING, period MEANS TERM, AND time IS TIME. SO THE ANSWER IS EITHER $c$ OR $d$. period, take period, WE DON'T SAY take period, SO THE ANSWER IS time."

[ID17 (only the latter part of the protocol)]

"Friends like to be given something you've taken, you have taken time, take time, take a moment, IT'S moment. Take a moment and trouble to make for them. WELL, THIS SEEMS MORE DIFFICULT. WELL, morning IS NOT THE ANSWER. Morning IS NOT, but you've taken period, moment MEANS INSTANT, period MEANS TERM, time IS TIME. WELL, like to be something you've taken, take period, and trouble to make for them, take a period, THE ANSWER IS period? I DON'T KNOW. Trouble to make for them. WHO ARE THEM? You've taken... I DON'T KNOW. I DECIDE time IS THE ANSWER FOR NOW." 
Both of these participants tried to fill in the choices in the context, especially with take, located just before the brackets. Although ID17 did not give any reason for selecting time for the answer, ID7 explicitly said that "WE DON'T SAY take period." Actually, 13 participants out of 20 who thought aloud used the collocation with take as a clue for the answer, and use of collocation knowledge was the largest for this item (Mean $=2.54$ participants, $S D=3.25$ ) among the 39 items. Since there was only one item it is impossible to generalize the results, but one possibility is that a thinking-aloud condition has an advantage in identifying a collocation with a word in context. However, the overall results suggest the high possibility that the allotted time influenced the test performance more than the think aloud protocol did.

\section{Conclusion}

This study investigated the reactivity effect on Japanese EFL learners' performance in MC vocabulary tests. Comparing the results of the thinking aloud group and the non-thinking-aloud group, the difference in test performance approached significance, with the thinking aloud group obtaining the higher score. However, most of the items that had a significant difference were located in the last quarter of the test; hence, the reason for the difference was not related to the feature of thinking aloud but to the time taken for the test. This suggests that thinking aloud itself does not change the test performance in MC vocabulary tests, as was also revealed in the reading tests of Leow \& Morgan-Short (2004). Therefore, this study adds further proof of the validity of using thinking aloud as a methodology for collecting data about test-taking processes. However, using thinking aloud, especially introspective verbal reports, almost always increases the time taken for the task, so we need to consider this fact when interpreting the data. Also, this study suggests that thinking aloud is not suitable for tasks whose time allotment is short, such as scanning and skimming in reading tests. Further research should give participants in both groups as much time as they want to complete the tasks, and thus minimize the time effect. Such research will clarify the validity of thinking aloud more and contribute generally to research using thinking aloud.

Note.

${ }^{1}$ A capitalized protocol was produced in Japanese, following Nikolov (2007).

This paper was based on the presentation in 2008 at the conference of the 1st JACET Joint Research Forum on English Vocabulary and Reading at Kwansei Gakuin University. 


\section{References}

Biggs, S. F., Rosman, A. J., \& Serfenian, G. K. (1993). Methodological issues in judgment and decision-making research: Concurrent verbal protocol validity and simultaneous traces of process data. Journal of Behavioral Decision Making, 9, 234-248.

Block, E. L. (1986). The comprehension strategies of second language readers. TESOL Quarterly, 20, 463-494.

Bond, T. G., \& Fox, C. M. (2007). Applying the Rasch model. Mahwah, NJ: Lawrence Erlbaum.

Brown, A. L., \& Day, J. D. (1983). Macrorules for summarizing texts: The development of expertise. Journal of Verbal Learning and Verbal Behavior, 22, 1-14.

Cain, K. (2007). Deriving word meanings from context: Does explanation facilitate contextual analysis? Journal of Research in Reading, 30, 347-359.

Cohen, A. D., \& Upton, T. A. (2007). 'I want to go back to the text': Response strategies on the reading subtest of the new TOEFL®. Language Testing, 24, 209-250.

Ellis, R. (1994). The study of second language acquisition. Oxford University Press.

Glass, G. V., \& Hopkins, K. D. (1996). Statistical methods in education and psychology. MA: Allyn \& Bacon.

Kavale, K., \& Schreiner, R. (1979). The reading process of above average and average readers: A comparison of the use of reasoning strategies in responding to standardized comprehension measures. Reading Research Quarterly, 15, 102-128.

Kern, R. (1989). Second language reading strategy instruction: Its effects on comprehension and word inference ability. Modern Language Journal, 73, 135-149.

Leow, R. P., \& Morgan-Short, K. (2004). To think aloud or not to think aloud: The issue of reactivity in SLA research methodology. Studies in Second Language Acquisition, 26, 35-57.

Linacre, M. (2006) Winsteps [Computer software]. Chicago, IL: Winsteps.

Nikolov, M. (2006). Test-taking strategies of 12- and 13-year-old Hungarian learners of EFL: Why whales have migraines. Language Learning, 56, 1-51.

Russo, L. E., Johnson, E. J., \& Stephens, D. (1989). The validity of verbal protocols. Memory and Cognition, 17, 759-769.

Takac, V. P. (2008). Vocabulary learning strategies and foreign language acquisition. Great Britain: Cromwell. 
Appendix

Examples of Experimental Tests (* indicate correct answers)

She waved her ( $\quad$ ) in front of her and she said: My daughter wrote the best art book ever written.
a. ability
b. hand*
c. power
d. skill

The bottles you buy today contain rain (

) which fell up to 80 years ago.
a. forest
b. sky
c. window
d. water*

She could ( ) his face quite clearly, as it was lit by the gas street lamp just a few feet behind him.
a. buy
b. develop
c. fall
d. see*

Each child's home ( $)$ is different, and not all children will want to talk about it in school.
a. circumstance*
b. competition
c. length
d. whisper

A common public ( $)$ is that the Aquino government is doing something that goes against the time-honored Filipino tradition and cultural values.
a. aspect
b. gaze
c. perception
d. survey 